\title{
A three-dimensional anisotropic point process characterization for pharmaceutical coatings
}

Article in Spatial Statistics available online June 2017 under https://doi.org/10.1016/j.spasta.2017.05.003

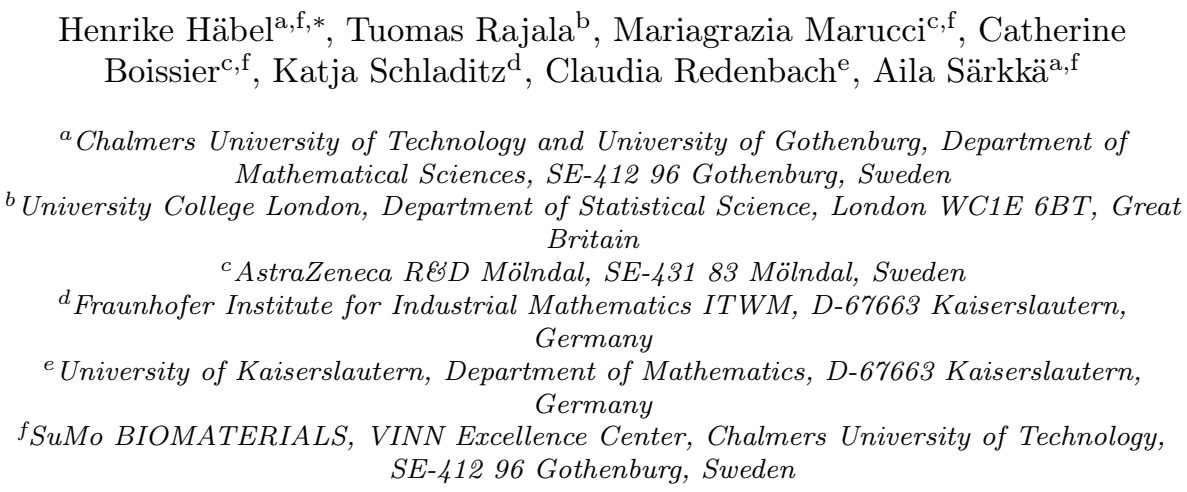

Henrike Häbel ${ }^{\mathrm{a}, \mathrm{f}, *}$, Tuomas Rajala ${ }^{\mathrm{b}}$, Mariagrazia Marucci ${ }^{\mathrm{c}, \mathrm{f}}$, Catherine Boissier $^{c, f}$, Katja Schladitz ${ }^{\mathrm{d}}$, Claudia Redenbach ${ }^{\mathrm{e}}$, Aila Särkkäa,f

${ }^{a}$ Chalmers University of Technology and University of Gothenburg, Department of Mathematical Sciences, SE-41296 Gothenburg, Sweden

${ }^{b}$ University College London, Department of Statistical Science, London WC1E 6BT, Great Britain

${ }^{c}$ AstraZeneca RESD Mölndal, SE-431 83 Mölndal, Sweden

${ }^{d}$ Fraunhofer Institute for Industrial Mathematics ITWM, D-67663 Kaiserslautern, Germany

${ }^{e}$ University of Kaiserslautern, Department of Mathematics, D-67663 Kaiserslautern, Germany

${ }^{f}$ SuMo BIOMATERIALS, VINN Excellence Center, Chalmers University of Technology, SE-412 96 Gothenburg, Sweden

\begin{abstract}
Spatial characterization and modeling of the structure of a material may provide valuable knowledge on its properties and function. Especially, for a drug formulation coated with a polymer film, understanding the relationship between pore structure and drug release properties is important to optimize the coating film design. Here, we use methods from image analysis and spatial statistics to characterize and model the pore structure in pharmaceutical coatings. More precisely, we use and develop point process theory to characterize the branching structure of a polymer blended film with data from confocal laser scanning microscopy. Point patterns, extracted by identifying branching points of pore channels, are both inhomogeneous and anisotropic. Therefore, we introduce a directional version of the inhomogeneous $K$-function to study the anisotropy and then suggest two alternative ways to model the anisotropic
\end{abstract}

\footnotetext{
*Corresponding author

Email address: henrike.habel@chalmers.se (Henrike Häbel)
} 
three-dimensional branching point structure. First, we apply a linear transformation to the data such that it appears isotropic and subsequently fit isotropic inhomogeneous Strauss or Lennard-Jones models to the transformed pattern. Second, we include the anisotropy directly in a Lennard-Jones and a more flexible step-function model with anisotropic pair-potential functions. The methods presented here will be useful for anisotropic inhomogeneous point patterns in general and for characterizing pharmaceutical coatings or other porous material in particular.

Keywords: Inhomogeneity, $K$-function, Lennard-Jones pair-potential function, pairwise Gibbs process, porous media

\section{Introduction}

Characterization and understanding of the pore structure within pharmaceutical coatings is essential in order to control their mass transport properties like permeability (Siepmann et al., 2008). Pharmaceutical coatings or dosage films

5 are usually sprayed around drug formulations to achieve delayed, sustained, or repeated drug release (Wen and $\mathrm{Li}, 2010$ ). Two crucial factors affecting mass transport and overall releasability of a drug are pore connectivity and tortuosity (Siegel, 2012). These two characteristics can be studied by analyzing the number, location and connection of the pore branching points, where at least three pore channels meet. For example, the more branching points there are relative to the number of channel ends, the better connected the channels are (Häbel et al. 2016).

The dosage film studied here is a blended film of two cellulosic polymers, namely ethyl cellulose (EC) and hydroxypropyl cellulose (HPC). Such bio-based 15 films are non-toxic, non-allergenic, and have good film forming properties and stability (Marucci et al. 2009, Siepmann et al. 2008). In contrast to EC, HPC is soluble in water and may act as a pore former (Marucci et al., 2013). Hence, the connected HPC-rich phase can be referred to as the pore phase and the EC-rich phase as the solid phase. Previous studies have shown some indications 

anisotropic (Häbel et al., 2016, Marucci et al., 2013). In this work, we find statistical evidence for the inhomogeneity and anisotropy by describing the pore structure in terms of its branching points and by using methods from point process theory. As the first step towards drawing conclusions on mass transport the locations of the branching points. Special attention is paid to anisotropy as describing directional trends in the structure may help us to understand not only mass transport properties, but also the film forming mechanism. For this purpose, methods for analyzing anisotropic inhomogeneous three-dimensional point patterns are presented. The challenges are to describe the type of the anisotropy detected in the data and to clearly distinguish it from inhomogeneity.

In recent research, geometric anisotropy has been of great interest as it provides a rather simple model framework. A point process is assumed to be geometrically anisotropic if it can be represented as a linear transformation of bach et al. (2009); and Wong and Chiu (2016). Anisotropy may also refer to point processes, where the points tend to be located along lines. In order to detect and model anisotropy, spatial summary statistics of point pairs need to be functions of both length and orientation of pairwise difference vectors. The 40 $K$-function, describing the expected number of points within a certain distance, was generalized for the detection of linearities in (Møller et al. 2016). Instead of considering points in a ball, directed cylinders are used as structure elements. A directed double cone was introduced as an alternative structure element in Redenbach et al. (2009). In Safavimanesh and Redenbach (2016), the cylin-

45 drical $K$-function is compared to a conical alternative for three-dimensional compressed or columnar point patterns, where the conical $K$-function appeared more suitable for compressed point patterns.

Spatial directional trends may also occur in various other ways that cannot easily be described with regular shapes. Directional analyses and tests for isotropy have been discussed in the recent literature. For example, Guan et al. 
(2006) introduce an asymptotic $\chi^{2}$-test and Møller and Toftaker (2014); Rajala et al. (2016); and Wong and Chiu (2016) consider geometric anisotropy.

An isotropy test based on replicated data was suggested in Redenbach et al. (2009). Directional analyses can also be done by using wavelets (Mateu and Nicolis, 2012) or spectral theory (Møller and Toftaker, 2014, Mugglestone and Renshaw, 1996).

In this work, we conduct an orientational study of point pairs in order to find evidence for anisotropy in a given inhomogeneous point pattern. Based on a preliminary analysis, we suspect that distances between pore branching points tend to be smaller vertically than horizontally, which is typical for structures compressed vertically. That is why, a three-dimensional inhomogeneous version of the conical $K$-function is introduced. Furthermore, we try to find a model that describes the branching point structure. The goal of the model fitting is not only to characterize and understand the spatial arrangement of the pore branching points, but also to explain the physical chemical dynamics underlying their formation. That is why we use the model family of finite pairwise interaction Gibbs processes, which allows for interaction between points and links back to statistical mechanics. When studying the interaction between two molecules, attractive and repulsive forces are often assumed and combined in a Lennard70 Jones potential as a function of the distance between the molecules (Zhen and Davies, 1983). Following the idea of intermolecular interaction, a Gibbs model with a Lennard-Jones pair-potential function seems a reasonable first choice. The Lennard-Jones model is compared to the Strauss model with only inhibition between points and a generalization of the Strauss model, namely a step-function model, allowing for attraction or inhibition at several ranges.

We suggest two versions of anisotropic pairwise interaction Gibbs processes. In the first approach similar to Wong and Chiu (2016), we assume geometric anisotropy and apply a linear transformation to obtain an almost isotropic point pattern. Then, an isotropic and inhomogeneous model is fitted to the transformed point pattern. Models for the original, untransformed point pattern are obtained by transforming the fitted model to an anisotropic one by the 
inverse of the operation of the previous step. In a new second approach, an anisotropic and inhomogeneous model is directly fitted to the data without any transformations.

85 To our knowledge, Gibbs point process models with anisotropic pair-potential functions have not yet been studied for three-dimensional point patterns and without assuming geometric anisotropy. We show the usefulness of anisotropic pair-potential functions for characterizing and modeling inhomogeneous and anisotropic structures on the example of a porous polymer blended film. We present a simple and efficient methodology that is general enough to be applicable to various other point patterns.

\section{Material}

\subsection{Preparation of polymer films}

The porous films prepared for this study are composed of two cellulose derivatives, namely $70 \%$ (w/w, dry basis) ethyl cellulose (EC Ethocel ${ }^{\mathrm{TM}}$ Standard Premium of viscosity grade $10 \mathrm{cP}$, Dow Wolff Cellulosics GmbH, Germany) and $30 \%$ (w/w, dry basis) hydroxypropyl cellulose (HPC, grade LF, Aqualon, USA). $30 \%$ HPC was used in order to obtain a connected, percolating pore phase with channels going from one film side to the other (Marucci et al. 2009). 6\% of the HPC had been fluorescent dye $(0.005 \mathrm{~m} / \mathrm{m}$ of glucose) labeled in preparation for the confocal laser scanning microscopy (CLSM, Nikon D-ECLIPSE C1 confocal system with Eclipse TE2000-E inverted microscope). This was done in order to achieve a good contrast between HPC and EC.

The two polymers were dissolved in hydrous ethanol $(95 \% \mathrm{v} / \mathrm{v}$, Kemetyl $\mathrm{AB}$, Sweden) at room temperature under stirring overnight. For manufacturing a free film, the polymer solution was sprayed onto a rotating cylindrical Teflon drum with a moving atomizer nozzle in a modified fluidized-bed chamber at AstraZeneca R\&D Mölndal, Sweden, following the procedures as described by Gebäck et al. (2015). While the film layers dry, ethanol evaporation induces 110 phase separation of EC and HPC resulting in EC-rich and HPC-rich phases. 
The process is frozen by a high film viscosity which is reached at a certain solvent concentration (Marucci et al., 2013). Since several coating layers are sprayed on top of each other, the lower layers are re-wetted more often than upper layers. Consequently and due to a small increase in temperature at the end of the spraying process, phase separation occurs to a lower extent in the upper layers resulting in smaller domains towards the air-side of the film compared to the drum-side (Gebäck et al., 2015; Marucci et al. 2013). As the interfacial free energy is minimized during phase separation, new HPC drops are more likely to coalesce with large HPC domains than smaller ones. This may result in elongated HPC-rich channels. Due to the effects of the film manufacturing, the pore structure is relatively isotropic and homogeneous in each sprayed layer, but anisotropic and inhomogeneous in the air-to-drum-side direction along the film depth (Gebäck et al., 2015, Häbel et al. 2016).

\subsection{Data sets}

In the present work, a small sample from the center part of a dried film was imaged using CLSM in order to visualize the three dimensional pore structure. The $\mathrm{x}-\mathrm{y}$ pixel size on each two-dimensional layer was $78.57 \times 78.5 \mathrm{~nm}^{2}$ and the acquiring step size through the film from air-side to drum-side was $100 \mathrm{~nm}$. Furthermore, the cross-section of the film was imaged by scanning electron microscopy (SEM Quante 200, FEI Company). For this purpose, a piece of the film was embedded in an epoxy-glue mold and the resulting stem of glue was cut in a Leica Ultracut UCT ultramicrotome to expose the film cross-sections. First a glass knife, then a diamond knife was used for the cutting. The embedded cut film was exposed to water for several days to remove all leachable HPC. The cross-section was coated with a thin layer of gold in an ion-sputtering device (Cressington sputter coater 108 ants) before imaging.

Figure 1 presents a small section of the obtained three-dimensional CLSM data on the left. The HPC-rich phase, which corresponds to the pore phase, is depicted in brighter voxels. The pores seem to be larger in cross-sections (xz, yz) than on the top layer (xy). Similar images showed that the domains on xy-layers 
at any film depth appear to form more homogeneous structures than on crosssections. Figure 1 shows a two-dimensional SEM image of the film cross-section with a higher resolution than of the CLSM image. It can be seen that there tend to be more, but smaller pores at the top layer (air-side) of the film than in the lower layers towards the drum-side. The pores appear to be elongated along the drum-side with vertical branches. Taking the differences in size and shape of the pores into account, the pore structure appears inhomogeneous and anisotropic. We find statistical evidence for this conclusion in the characterization presented in Section 4.1
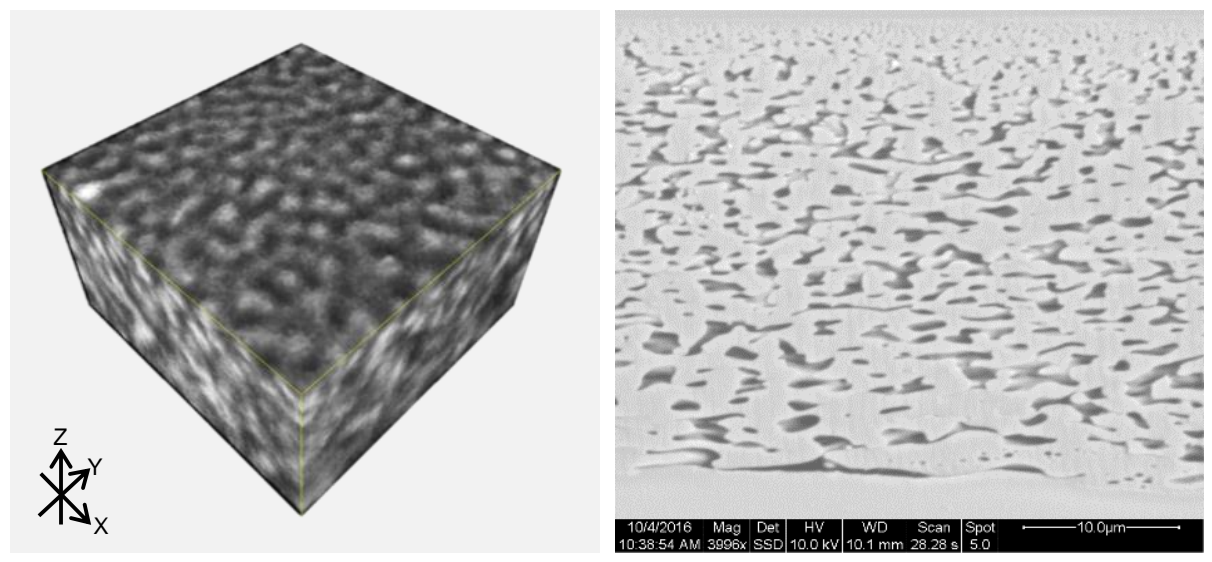

Figure 1: Left: Processed CLSM image of a $23.57 \times 23.57 \times 17 \mu \mathrm{m}^{3}$ film section. Dark voxels correspond to the EC-rich phase and bright voxels to the HPC-rich phase. Dimension z goes from drum-side to air-side. The pore structure appears anisotropic with large elongated domains in cross-sections $(\mathrm{z})$ compared to the displayed top layer (xy) with smaller domains forming a homogeneous structure. Right: Film cross-section (ca. $30 \mu \mathrm{m}$ ) obtained by SEM after HPC leaching showing more pores on the top (air-side) than on the bottom (drumside). The gray region above and below the pores is the epoxy-glue in which the sample was embedded.

\subsection{Image analysis}

The image processing for the pore branching point extraction was conducted in MAVI (MAVI - Modular algorithms for volume images, 2015). The aim of the image processing was to segment the pore (HPC-rich) and solid (EC-rich) 
phase. The major challenge was to develop a procedure that preserves the pore connectivity, while holding the volume fraction at the known level for $30 \% \mathrm{HPC}$ films. A three-dimensional ultimate homotopic skeleton was extracted from the binarized CLSM image following a topology preserving algorithm developed by Couprie et al. (2007). Here, a skeleton refers to a system of connected onedimensional line segments located centrally inside the pore phase. A voxel on the skeleton has value one and voxels outside the skeleton value zero. For a correction of edge effects introduced by the skeletonization method, the window was reduced by two voxel layers in all directions. A more detailed description of the image analysis methods used for noise reduction, binarization and skeletonization can be found in the supplementary material.

Isolated, end, and branching points of the skeleton were identified using a classification by neighbor counting in a 26 -neighborhood implemented in a function in R. For this purpose, the skeleton was translated into a spatial graph using the R package spatgraphs (Rajala, 2012), where each skeleton voxel corresponds to a node in the graph. For each node or skeleton voxel, the sum of the connected components is calculated. The obtained sum can be used to classify skeleton voxels into four classes.

0) If the sum is zero, the skeleton voxel is an isolated point with no neighbors. These points are important for the pore volume fraction, but they do not affect the mass transport. Consequently, isolated points were disregarded in the spatial modeling described below.

1) If the sum is one, the voxel is an end point with just one skeleton neighbor. A large number of end points may affect the mass transport as mass can get trapped in pore channels with dead ends. Here, however, the number of end points was very low throughout the whole film and end points were also declared irrelevant for the modeling.

2) If the sum is two, a voxel has two neighbors and is regarded as a connecting point. The connecting points can be used to study pore tortuosity and connectivity. For such studies, it is important to keep in mind, that the 
number and position of connecting skeleton points highly depends on the applied image analysis. That is why, we do not construct a model based on the whole skeleton, but only use branching points as they are more robust to the processing.

3) If the sum is larger than two, the voxel is classified as a branching point with at least three neighbors. Sets of branching points with maximal distance of three voxels between points were reduced to their centroid to make the branching point extraction more robust to structural peculiarities of a sample and image processing choices (Liebscher and Redenbach, 2013).

The graph was reduced to the branching points by deleting all other nodes from the graph that were not branching points. Less than $10 \%$ of the remaining branching points are connected to more than three other branching points.

\section{Methods and Theory}

The set of pore branching point locations extracted from the processed CLSM images of the film form a point pattern, which is mathematically expressed as a realization of a point process $X=\left\{X_{i}\right\}$ of random locations

$$
X_{i}: \Omega \rightarrow \mathbb{R}^{3}, i=1,2, \ldots
$$

on some probability space $(\Omega, \mathcal{A}, \mathbb{P})$. In what follows, $W \subset \mathbb{R}^{3}$ denotes the bounded observation window in which the point process $X$ is observed. Let $|W|$ denote the volume of the window and $N_{X}(W) \geq 0$ the number of points of $X$ in $W$. The first moment of the integer-valued random variable $N_{X}(W)$ is given by

$$
\mathbb{E}\left[N_{X}(W)\right]=\int_{W} \lambda(s) d s
$$

where it is assumed that $\mathbb{E}\left[N_{X}(W)\right]$ has a density $\lambda$ with respect to the Lebesque measure. $\lambda$ is called the first-order intensity function. For stationary and isotropic point processes with constant $\lambda(s)=\lambda, \forall s \in \mathbb{R}^{3}$, Ripley's $K$-function 
can be used to describe second-order characteristics, where $\lambda K(r)$ gives the expected number of points of $X$ within a ball $b(o, r)$ without counting $o$ itself given that there is a point at $o$ (Illian et al. 2008, p.214 ff. Chiu et al. 2013, p.99 ff.).

\subsection{Anisotropic and inhomogeneous $K$-function}

The $K$-function was generalized in Baddeley et al. (2000) for second-order intensity-reweighted stationary point processes, where second-order characteristics are still functions of distances between points. Combining this inhomogeneous version with the directional $K$-function presented in Redenbach et al. (2009), we introduce an anisotropic and inhomogeneous version of the $K$-function, $K_{a n i n}$.

Let $C(u, a)$ be a double cone with central axis $u \in S^{2}$ on the unit sphere $S^{2}$ and opening angle $2 a \in[0, \pi]$. For a ball $b(o, r)$ with radius $r$ around the origin $o$, let $C(u, a, r)=C(u, a) \cap b(o, r)$. Then we define

$$
K_{\text {anin }}(u, a, r)=\frac{1}{|W|} \mathbb{E} \sum_{x \in X \cap W} \sum_{y \neq x \in X \cap W} \frac{\mathbf{1}[(y-x) \in C(u, a, r)]}{\lambda(x) \lambda(y)}, \quad r \geq 0 .
$$

Figure 2 shows the structure element $C(u, a, r)$ for angle $a=\pi / 6$ in direction $u=(1,0,0)$ as the intersection of the double cone $C(u, a)=C(u, a, \infty)$ with the ball $b(o, r)$. The inhomogeneous version by Baddeley et al. (2000) is obtained for $a=\pi / 2$ and a constant $\lambda$ yields the conical $K$-function by Redenbach et al. 215 (2009).

An estimator for $K_{\text {anin }}$ is given by

$$
\widehat{K}_{\text {anin }}(u, a, r)=\frac{1}{|W|} \sum_{x \in X \cap W} \sum_{y \neq x \in X \cap W} \frac{\mathbf{1}[(y-x) \in C(u, a, r)]}{w_{x y} \hat{\lambda}(x) \hat{\lambda}(y)}, \quad r \geq 0
$$

with translation edge correction factors $w_{x y}=|(W-x) \cap(W-y)|$, where $W-x$ refers to the translation of the window $W$ by the vector $x$. The intensity function $\lambda$ was estimated using an edge corrected Gaussian kernel estimator, for which the smoothing bandwidth was chosen using the Cronie and van Lieshout 220 (2016) method. 


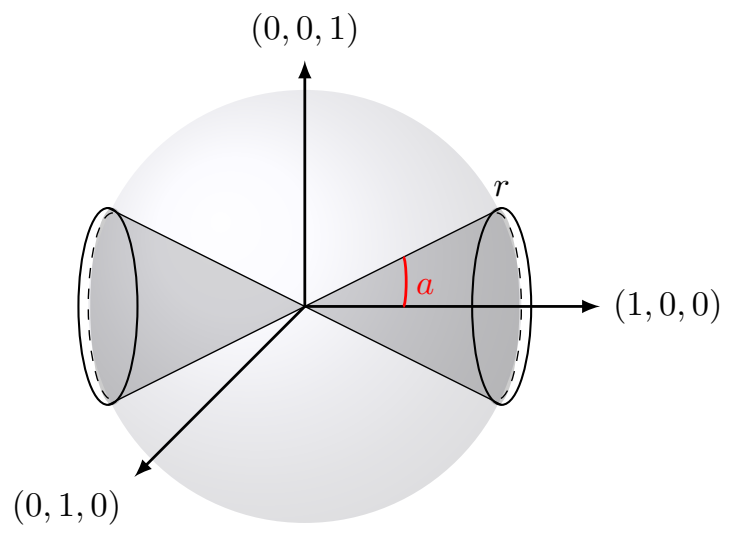

Figure 2: Structure element $C(u, a, r)$ (dark gray) of the directional $K$-function in direction $u=(1,0,0)$ with opening angle $2 a=2 \pi / 6$ in a ball $b(o, r)$ centered at the origin $o$ with radius $r$ (light gray).

\subsection{Finite pairwise interaction Gibbs processes}

Let us consider the spatial exponential family of finite Gibbs processes with $n$ points of $X$ in $W$ and density function

$$
f_{n}\left(x_{1}, \ldots, x_{n}\right)=\frac{\exp \left(-E\left(x_{1}, \ldots, x_{n}\right)\right)}{Z_{n}}
$$

for $x_{1}, \ldots, x_{n} \in W$, where $Z_{n}$ is a normalizing constant and the function $E$ : $\mathbb{R}^{n \times 3} \longrightarrow(-\infty, \infty]$ assigns an 'energy' to the point configuration $\left(x_{1}, \ldots, x_{n}\right)$ (Illian et al. 2008, p.141 ff.). For an isotropic pairwise interaction process, the energy function can be written in the following form

$$
E\left(x_{1}, \ldots, x_{n}\right)=\sum_{i} \alpha\left(x_{i}\right)+\sum_{i<j} \phi\left(d_{i j}\right)
$$

for pairwise distances $d_{i j}=\left\|x_{i}-x_{j}\right\|$. The function $\alpha: \mathbb{R}^{3} \longrightarrow \mathbb{R}$ is called the chemical activity function and describes the likelihood of a point being located at $x \in W$. For a stationary process, $\alpha(x)=\alpha, \alpha \in \mathbb{R}$, is a constant. The function describing pairwise interactions $\phi:(0, \infty) \longrightarrow \mathbb{R} \cup\{\infty\}$ is called the pair-potential function. We consider three different pair-potential functions. The first yields the Strauss process with pair-potential function

$$
\phi_{S}(r)=\beta \mathbf{1}[r<R]
$$


and serves as a reference model with interaction between points being less than distance $R>0$ apart. Each $R$-close point pair contributes with interaction strength $\gamma=\exp (-\beta), \beta \in \mathbb{R}, 0<\gamma<\infty$. A regular pattern with inhibition between points is obtained with $\beta>0$ and $\gamma<1$, respectively. $\beta=0$ and $\gamma=1$ correspond to a Poisson process with no interaction. For a process with a fixed number of points, $\beta<0$ and $\gamma>1$ yield attraction. Otherwise an attractive Strauss process is not well defined (Møller and Waagepetersen, 2004, p.84). The Strauss model can be generalized by a step pair-potential function

$$
\phi_{S}(r)= \begin{cases}\beta_{s} & , c_{s-1}<r \leq c_{s} \\ 0 & , r>c_{S}\end{cases}
$$

allowing for both inhibition $\left(\beta_{s}>0\right)$ and attraction $\left(\beta_{s}<0\right)$ at different ranges $c_{s}, s=1, \ldots, S$ with $c_{0}=0$. In that way, any continuous pair-potential function can be approximated by a sufficiently large number of jumps $S$ with relatively weak model assumptions (Clyde and Strauss, 1991). A popular parametric model for short range inhibition and mid-range attraction is the 12-6 LennardJones potential

$$
\phi_{L J}(r)=4 \epsilon\left(\left(\frac{\sigma}{r}\right)^{12}-\left(\frac{\sigma}{r}\right)^{6}\right) .
$$

The pairwise interaction changes from inhibition to attraction at the characteristic distance $\sigma>0$, where the pair-potential function becomes zero. $\sigma$ may also be referred to as an interaction range. The parameter $\epsilon \geq 0$ determines the interaction strength. A more general Lennard-Jones model can be found in Ogata and Tanemura (1981).

\subsection{Anisotropic pair-potential functions}

Anisotropic potentials have already been used for describing intermolecular dynamics with different interactions within and between connected components of a system (Affouard et al. 1996). Here, the idea is adopted to the pairpotential function of a pairwise interaction Gibbs process, where points may interact differently depending on the direction. 
Isotropic pair-potential functions as discussed in Section 3.2 assign the same interaction strength to point pairs with the same interpoint distance. We now introduce anisotropic pair-potential functions, where the interaction parameters depend on both the length and the orientation of the pairwise difference vectors. In particular, for direction $u \in S^{2}$ and angle $a \in[0, \pi / 2]$, point pairs with a difference vector inside a double cone $C(u, a)$ are allowed to have different model parameter values than point pairs with difference vectors outside the double cone. Let $v=y-x$ be the difference vector of a point pair $(x, y)$ of $X \cap W$ and let $\delta_{(u, a)}(v)=\mathbf{1}[v \in C(u, a)]$. Then (2) and $[3$ can be modified to

$$
\phi_{S}(u, a, v)= \begin{cases}\beta_{1_{s}} & , c_{s-1}<r \leq c_{s}, \delta_{(u, a)}(v)=1 \\ \beta_{2_{s}} & , c_{s-1}<r \leq c_{s}, \delta_{(u, a)}(v)=0 \\ 0 & , r>c_{S}\end{cases}
$$

and

$$
\begin{aligned}
\phi_{L J}(u, a, v)=\delta_{(u, a)}(v) 4 \epsilon_{1}\left(\left(\frac{\sigma_{1}}{\|v\|}\right)^{12}-\left(\frac{\sigma_{1}}{\|v\|}\right)^{6}\right) \\
+\left(1-\delta_{(u, a)}(v)\right) 4 \epsilon_{2}\left(\left(\frac{\sigma_{2}}{\|v\|}\right)^{12}-\left(\frac{\sigma_{2}}{\|v\|}\right)^{6}\right)
\end{aligned}
$$

In the pair-potential function (4), $\beta_{1_{s}}$ and $\beta_{2 s}, s=1, \ldots, S$, are interaction strength parameters inside the double cone and outside the double cone, respectively. The corresponding ranges of interaction are here chosen to be the same in all directions. In $\sqrt[5]{5}, \epsilon_{1}$ and $\epsilon_{2}$ are the interaction strength parameters of the Lennard-Jones model in the double cone and outside the double cone, respectively, $\sigma_{1}$ and $\sigma_{2}$ are the corresponding interaction ranges. Besides single double cones also combinations of double cones or other structuring elements may be used to define anisotropic pair-potential functions.

\subsection{Parameter estimation and simulations}

There are several methods available for the parameter estimation of finite Gibbs processes with pairwise interactions. Comparisons of different methods

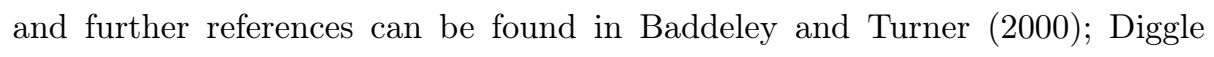



regression version of the maximum pseudolikelihood estimation as discussed in Baddeley et al. (2014). This method is based on the Papangelou conditional intensity such that the normalizing constant can be disregarded. Furthermore, it is computationally simple because it can be computed with standard software. technique as suggested in Heikkinen and Penttinen (1999) was used since it can handle large variations of parameter estimates due to low point counts in the design matrix. Minus-sampling was chosen as edge correction (Illian et al., 2008 p.185 f.).

Markov chain Monte Carlo was used for the simulation of point patterns. In general, Metropolis-Hastings algorithms are popular for the simulation of Gibbs processes with an unnormalized density on a bounded region (Møller and Waagepetersen, 2004, p.107). Here, we used the Metropolis-Hastings algorithm for the conditional case of point processes with a density presented in (Møller and Waagepetersen, 2004, p.109). We ran $2 \cdot 10^{5}$ iterations on a standard 2016 laptop (16Gb RAM, $2.4 \mathrm{GHz}$ processor) to make sure that the stationary distribution was reached, which took about 14 minutes. A guard window as discussed in Mateu and Montes (2001) was used to correct for missing data outside the observation window with a fixed number of points resulting in the same intensity in the original window. The guard window size was chosen to have an additional side length of four times the interaction range. As the interaction range is theoretically infinite for the Lennard-Jones potential, the interpoint distances were truncated to obtain a finite range of $4 \sigma$ and $4 \max \left(\sigma_{1}, \sigma_{2}\right)$, respectively.

\section{Results}

The spatial analysis was conducted in $\mathrm{R}$ version 3.3.1. The anisotropic and inhomogeneous $K$-function can be found in the package Kdirectional available on Github. The newly implemented R-functions are extensions of functions available in the $\mathrm{R}$ package spatstat version 1.37-0 (Baddeley and Turner, 2005). 
The main difference in the new R-functions is the construction of the design matrix. Otherwise, the new functions call the standard $g \operatorname{lm}()$ R-function and the package vblogistic available on Github. For the Lennard-Jones model, interpoint distances were rescaled to a unit equal to the smallest interpoint distance present in the point pattern to reduce numerical problems such as slow convergence (Baddeley and Dereudre, 2013).

Two samples were available for the analysis, which gave similar results and let to the same conclusions. In what follows, the results of one sample are presented in detail. Corresponding plots of the other sample can be found in the supplementary material.

\subsection{Pore characterization}

Inhomogeneity in the studied data is visible in the histogram of the number of branching points per film layer shown in Figure 3 (top left). The intensity within the pore branching point pattern seems to follow a linear trend along the film depth. This result can be explained by the film manufacturing. The number of connecting points between two branching points tends to be comparable to their Euclidean distance. This finding speaks for rather straight pore channels. In what follows, we will focus on the pore channel orientation.

For detecting anisotropy, the orientation of pairwise difference vectors was investigated. Studying the inclination is of special interest as it can be linked to mass transport properties. If a pore channel is close to a straight, vertical cylindrical tube, for instance, the mass transport will only be affected by the pore thickness.

Figure 3 (top right) shows the inclination angles of the pairwise distance vectors between connected pore branching points. Interestingly, the distribution of the inclination for the first nearest neighbors is similar as can be seen in Figure 3 (bottom left). Judging by the peaks of the bimodal distributions, it appears that a point pair has a preferred orientation. This observation can be due to the fact that the points lie on a gird. However, jittering and randomizing the order of the points still resulted in a bimodal distribution which can be used as evidence 
for anisotropy in the point pattern. Furthermore, it can be concluded that the This is an important finding for describing the pore tortuosity since it indicates that the shortest pore channels are mostly close to vertical. The anisotropic behavior as observed for the connected branching points and the nearest neighbors seems to decrease for the third nearest neighbor whose inclination distribution is approaching the isotropic case as shown in Figure 3 (bottom right). In other words, the orientation of pairwise difference vectors seems to depend on their length, where short difference vectors are rather vertically and long ones more horizontally oriented. This is another sign for anisotropy.

In order to find further evidence for anisotropy, the anisotropic and inhomogeneous version of the $K$-function, $K_{\text {anin }}$, can be studied in Figure 4 in form of its variant

$$
L(r)=\sqrt[3]{\frac{4 K(r)}{3 \pi}}, \quad r \geq 0 .
$$

Figure 4 also shows estimates for the inhomogeneous and conical $K$-functions. of the pore branching point extraction algorithm. Furthermore, there seem to be more branching points within short distance $r>0.03$ than expected in a completely spatially random pattern without interactions between points (black dotted line). The point pattern appears clustered even for longer distances when not accounting for anisotropy in Lest inhomo $_{\text {. }}$

For the directional analysis, an opening angle of $\pi / 3$ was chosen in order to distinguish between the chosen direction by non-overlapping double cones and still have wide enough cones containing sufficiently many points for a good estimation of the $K$-function. Varying the opening angle lead to the same conclu325 sions only more or less prominent for smaller or wider angles. When studying the $K$-function in different directions, the interaction in the z-direction along film depth appears to be different from the other two main directions $(\mathrm{x}=(1,0,0)$ and $\mathrm{y}=(0,1,0))$. Whereas there is attraction between points in z-direction, the points form a rather regular pattern in $\mathrm{x}$ - and $\mathrm{y}$-directions. Consequently, inter- 

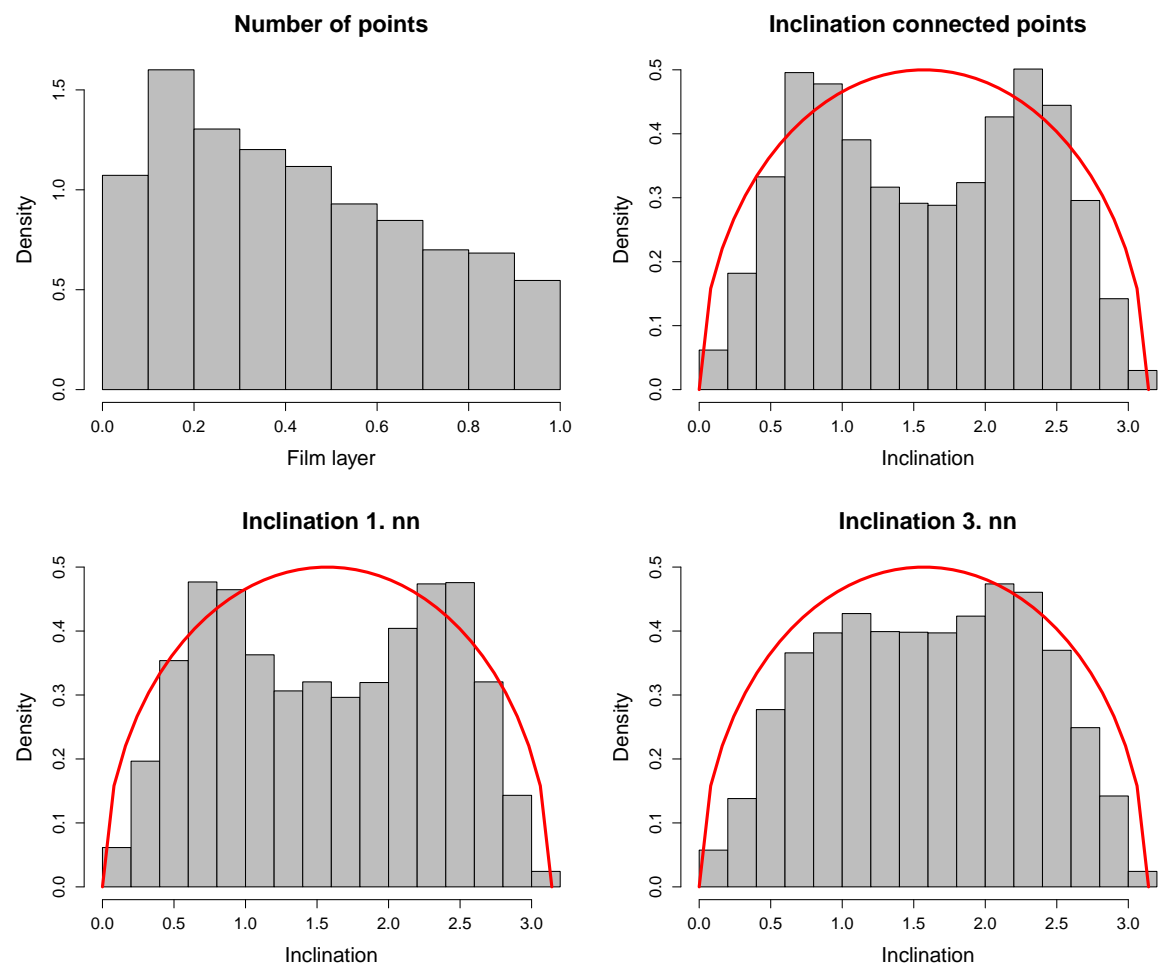

Figure 3: Top left: histogram of the number of branching points per film layer along film depth going from air-side (0) to drum-side (1). Top right: histogram of inclination of connected branching points showing preferred directions of about $\pi / 4$ and $3 \pi / 4$ radians from the $z$ axis in direction $(0,0,1)$. Bottom left: histogram of inclination of the first nearest neighbor. Bottom right: histogram of inclination of the third nearest neighbor. The inclination for an isotropic point pattern is represented by the solid curve.

point distances tend to be smaller vertically than horizontally and anisotropy in form of a directional trend can be detected.

\subsection{Pore branching point modeling}

Using the results from the pore characterization, four Gibbs models were fitted to the data, namely a compressed inhomogeneous Strauss (Strauss), a compressed inhomogeneous Lennard-Jones (LJ A) and two anisotropic Gibbs models with an anisotropic Lennard-Jones (LJ B) and step (Step) pair-potential 

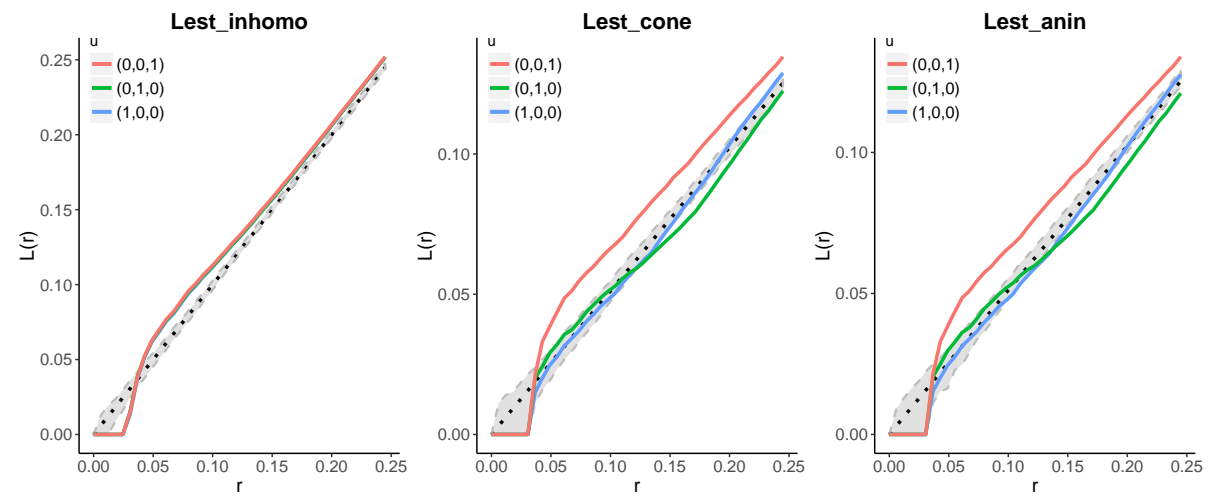

Figure 4: From left to right: estimated inhomogeneous $L$-function (Lest inhomo $_{\text {o }} a=\pi / 2$ ), conical $L$-function (Lest cone,$a=\pi / 6$ ), and anisotropic and inhomogeneous $L$-function (Lestanin,$a=\pi / 6$ ) for the pore branching points (data). The theoretical curve is given by $L_{\text {theo }}(u, a, r)=2 / 3 r^{3}(1-\cos (2 a))$ for a completely spatially random pattern and envelopes based on 999 simulations of the theoretical model are given.

function. Prior to fitting inhomogeneous Strauss and Lennard-Jones models with a linear trend in intensity along the film depth, the data were stretched to an isotropic pattern by a factor of $1 / 0.17=5.882$. This transformation factor was estimated by following the approach introduced in Redenbach et al. (2009), and among several compression factors the one resulting in the most isotropic pattern is identified using the $K$-function. The range parameter $R=0.1$ of the Strauss model was chosen through a grid search. The final model for the untransformed data is a by a factor of 0.17 compressed version of the fitted model. All linear transformations were done conserving the total volume of the observation window. For the anisotropic Lennard-Jones model, LJ B, the direction of the double cone was set to $\mathrm{z}=(0,0,1)$ and the opening angle to $2 \pi / 3$ based on a goodness-of-fit analysis over several angles. The corresponding estimated parameters can be found in Table 1 .

An anisotropic Strauss model was not fitted to the data as it appeared unsuitable for modeling the clustering observed along the film depth. Instead, its generalized version was fitted as a second anisotropic model, Step. At first, 10 equally spaced jump points were used in order to identify a smaller number 
of jumps for the final model. Several options were compared in a goodness355 of-fit analysis. The parameter estimates $\left(\hat{\gamma}_{2}=\exp \left(-\hat{\beta}_{2}\right)\right)$ for the first jump after the hard-core distance $d_{h c}=0.030$ are presented in Table 1 All other parameter estimates $\left(\hat{\beta}_{s}, s=1,3,4,5\right)$ for five jumps at distances $2 d_{h c}, \ldots, 5 d_{h c}$ are depicted in Figure 5

Global envelopes were constructed for the $L$-function and the rank envelope test introduced by Myllymäki et al. (2017) was conducted for each tested main direction. Figure 6 presents the respective summary plots for the directions $\mathrm{x}$ $=(1,0,0), \mathrm{y}=(0,1,0)$ and $\mathrm{z}=(0,0,1)$ and global envelopes based on 2499 simulations for a $95 \%$ confidence test.

\begin{tabular}{|c|c|c|c|c|c|c|c|c|}
\hline \multirow[t]{2}{*}{ Model } & \multicolumn{5}{|c|}{ Parameter estimates } & \multicolumn{3}{|c|}{$\mathrm{P}$-values } \\
\hline & \multicolumn{2}{|c|}{ range } & \multicolumn{2}{|c|}{ strength } & trend & \multirow[t]{2}{*}{$\mathrm{x}$} & \multirow[t]{2}{*}{$\mathrm{y}$} & \multirow[t]{2}{*}{$\mathrm{z}$} \\
\hline & $\mathrm{R}$ & & $\gamma$ & & & & & \\
\hline \multirow[t]{2}{*}{ Strauss } & 0.100 & & 0.802 & & -0.203 & 0.018 & 0.017 & 0.015 \\
\hline & $\sigma_{1}$ & $\sigma_{2}$ & $\epsilon_{1}$ & $\epsilon_{2}$ & & & & \\
\hline LJ A & 0.012 & & 1.577 & & -0.199 & 0.020 & 0.019 & 0.017 \\
\hline \multirow[t]{2}{*}{ LJ B } & 0.031 & 0.030 & 2.464 & 0.952 & -1.245 & 0.020 & 0.021 & 0.020 \\
\hline & $c_{1}$ & $c_{1}$ & $\gamma_{12}$ & $\gamma_{22}$ & & & & \\
\hline Step & 0.030 & 0.030 & 3.512 & 1.432 & -1.187 & 0.018 & 0.022 & 0.105 \\
\hline
\end{tabular}

Table 1: Parameter estimates for the four fitted models. Model Strauss is a compressed inhomogeneous Strauss model with interaction range $R$ and interaction strength $\gamma=\exp (-\beta)$. Model LJ A is a compressed inhomogeneous Gibbs model with a Lennard-Jones pair-potential function with interaction range $\sigma$ and interaction strength $\epsilon$. Model LJ B has an anisotropic Lennard-Jones pair-potential function with different parameter values $\left(\sigma_{1}, \epsilon_{1}\right)$ and $\left(\sigma_{2}, \epsilon_{2}\right)$ for point pairs within the structure element and outside. Model Step is a Gibbs model with an anisotropic step pair-potential function, where only the first jump after the hard-core distance is given for inside $\left(\gamma_{1_{2}}=\exp \left(-\beta_{1_{2}}\right)\right)$ and outside $\left(\gamma_{2} 2=\exp \left(-\beta_{2_{2}}\right)\right)$ the structure element. The slope estimates of the fitted linear trend along the film depth are listed under trend. Multiplicity adjusted p-values (Bonferroni correction) based on rank envelope tests with 2499 simulations are given for $L$-functions in three main directions $\mathrm{x}=(1,0,0), \mathrm{y}=(0,1,0)$ and $\mathrm{z}$ $=(0,0,1)$ estimated within a double cone with an opening angle of $\pi / 3$. 


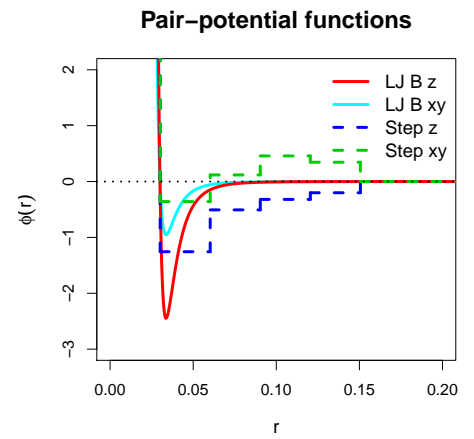

Figure 5: Pair-potential functions of the fitted anisotropic models LJ B and Step with different parameters inside a double cone with an opening angle of $2 \pi / 3$ in $\mathrm{z}=(0,0,1)$ direction than outside (xy).

\section{Discussion}

From Figure 6 and the p-values presented in Table 1, it can be concluded that the data do not seem to be compatible with any of the suggested models in all directions. Especially, the compressed models performed poorly. On one hand, this indicates that the geometrical anisotropy assumption might not hold and the observed directional trends have to be explained differently. Furthermore, the anisotropy study in Section 4.1 does not support a simple compression in zdirection, but suggests a more complex transformation. On the other hand, Ellis (1986) found that a very large compression may cause the transformed pattern to behave approximately like a Poisson process. This may also have happened here, when the data were stretched by a factor of $1 / 0.17$. In that case, parameters almost corresponding to the completely spatially random case are fitted and linear transformations become ineffective. This explanation is supported by the parameter estimate for the interaction strength $\hat{\gamma}=\exp (-\hat{\beta})=0.802$ of the Strauss model for the transformed data.

Fitting a Gibbs model with an anisotropic pair-potential function circumvents the problem of reaching Poisson like behavior. For the two fitted anisotropic models, in fact, a difference between the envelopes for the $\mathrm{x}$ - and $\mathrm{y}$-directions to 

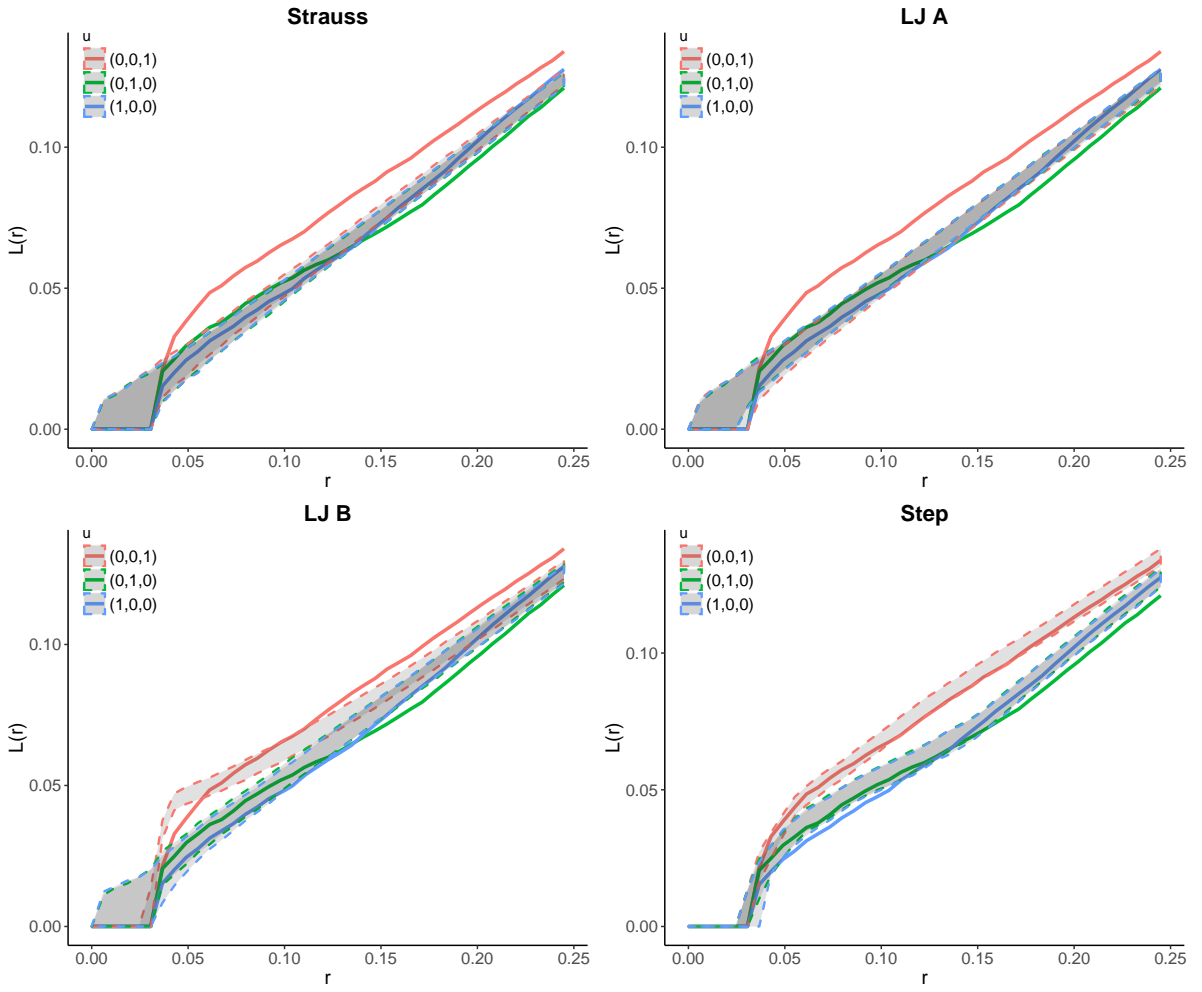

Figure 6: Anisotropic and inhomogeneous $L$-functions with global envelopes for the four fitted models, a compressed inhomogeneous Strauss (Strauss), a compressed inhomogeneous Lennard-Jones (LJ A), an anisotropic Lennard-Jones model (LJ B) and an anisotropic Gibbs model with a step pair-potential function (Step). The dashed lines give the global envelope bounds based on 2499 simulations. The thick solid curves correspond to the data. Three directions were investigated, $x=(1,0,0), y=(0,1,0)$ and $z=(0,0,1)$ within a double cone with opening angle $\pi / 3$. 
the z-direction can be seen in Figure 6. Model LJ B, performs well for interpoint distances up to 0.12 (about $2 \mu \mathrm{m}$ ) in $\mathrm{x}$ - and $\mathrm{y}$-directions and tends to overestimate the interaction strength for larger distances. Even though the model fit 385 is far from perfect according to the rank envelope test, the global envelopes for Model LJ B suggest that the model is not unfeasible. In z-direction for short interpoint distances up to 0.06 (about $1 \mu \mathrm{m}$ ), Model LJ B overestimates the interaction strength and tends to slightly underestimate it for larger distances. Changing the opening angle and structure element did not improve the model fit. The findings suggest that the model could be improved by also introducing distance dependent interaction parameters. For this purpose, the step pairpotential function was fitted, which captured the anisotropy in z-direction very well.

On studying the pair-potential functions of Model LJ B and Model Step in Figure 5, two explanations can be found on why the Lennard-Jones pairpotential function did not fit well. First, there seems to be mid-range repulsion in xy-direction which is not possible to capture in a Lennard-Jones pair-potential function. Second, even though the shape of the Lennard-Jones pair-potential function seems suitable in z-direction, the estimated attractive 400 range and strength were too low. From both anisotropic models two important conclusions can be drawn. First, the interaction range appears to be invariant under rotation. Second, the pairwise interaction seems to be more than twice as strong vertically along the film depth (z) than horizontally on each layer (xy). The model fit was very good in the $\mathrm{z}$-direction but not in the $\mathrm{x}$ - and $\mathrm{y}$-directions. The physical-chemical assumption was that the xy-layers are homogeneous and isotropic. No strong evidence against this assumption was found in the pore characterization analysis. Based on the goodness-of-fit results, however, one could reinvestigate this assumption. 


\section{Conclusions}

410 three-dimensional, inhomogeneous point patterns have been presented. For this purpose, new R-functions had to be implemented for the three-dimensional orientational analysis, model parameter estimation and simulations. A modeling approach based on a linear transformation of the point pattern was compared

415 to a model with an anisotropic pair-potential function. We have found that even though geometric anisotropy may be a good first guess, a model with an anisotropic pair-potential function might be the better choice.

The presented methods were applied to model the locations of pore branching points in a porous film used as a pharmaceutical coating. A preliminary model with an anisotropic Lennard-Jones pair-potential function motivated by molecular dynamics was given. The model was improved by changing the pairpotential function to a step-function allowing for inhibition and attraction at longer ranges. The model fit may be improved further by taking possible heterogeneity on film layers into account. In order to fit a representative model more replicates are needed, which are however very time consuming to obtain. A completely different modeling approach may also be interesting to study, where the pore branching points are modeled in consecutive layers. Such a model would describe more the temporal process of the film formation and less the molecular dynamics within the whole film.

Three important conclusions on the film pore structure were drawn. First, it was found that the number of branching points decreases linearly from upper to lower film layers. Second, the pore branching points appear to be connected to their nearest neighbor by almost vertical pore channels. Third, the vertical pairwise interaction between branching points seems to be different from horizontal interaction. These results are not only important for identifying important features of the pore structure, but also for further studies in which a complete model for the pore structure will be constructed.

This work has shown that it is possible to link experimental and statis- 
tical findings for a better understanding of anisotropy and inhomogeneity in three-dimensional data. The developed tools can be of great value for the characterization and modeling of any porous material using point process theory. In this way, material properties can be improved or new materials developed based on predictive science and fewer resource consuming experiments.

\section{Acknowledgment}

${ }_{445}$ This work is part of the VINN Excellence Centre SuMo BIOMATERIALS and has mainly been financed by the Swedish Governmental Agency for Innovations Systems, VINNOVA. In addition, the financial support from the Knut and Alice Wallenberg Foundation, KAW, and the Swedish Foundation for Strategic Research, SSF, is highly appreciated. The authors would like to thank Mats Rudemo (Chalmers University of Technology) for valuable discussions and important contributions and Johan Arnehed (AstraZeneca R\&D Mölndal) is thanked for conducting CLSM and SEM studies. The authors also thank the reviewers for their valuable comments.

\section{References}

455 Affouard, F., Kröger, M., Hess, S., 1996. Molecular dynamics of model liquid crystals composed of semiflexible molecules. Phys. Rev. E 54 (5), 5178-5186.

Baddeley, A. J., Coeurjolly, J. F., Rubak, E., Waagepetersen, R., 2014. Logistic regression for spatial Gibbs point processes. Biometrika 101 (2), 377-392.

Baddeley, A. J., Dereudre, D., 2013. Variational estimators for the parameters of Gibbs point process models. Bernoulli 19 (3), 905-930.

Baddeley, A. J., Møller, J., Waagepetersen, R., 2000. Non- and Semi-parametric Estimation of Interaction in Inhomogeneous Point Patterns. Stat. Neerl. 54 (3), 329-350. 
Baddeley, A. J., Turner, R., 2000. Practical Maximum Pseudolikelihood for Spatial Point Patterns (with Discussion). Aust. N.Z. J. Statist. 42 (3), 283322.

Baddeley, A. J., Turner, R., 2005. spatstat: An R Package for Analyzing Spatial Point Patterns. J. Stat. Softw. 12 (6), 1-42.

Chiu, S. N., Stoyan, D., Kendall, W. S., Mecke, J., 2013. Stochastic Geometry and its Applications, 3rd Edition. John Wiley and Sons, Chichester.

Clyde, M., Strauss, D., 1991. Logistic Regression for Spatial Pair-Potential Models. In: Spatial Statistics and Imaging. Institute of Mathematical Statistics, Hayward, pp. 14-30.

Couprie, M., Coeurjolly, D., Zrour, R., 2007. Discrete bisector function and 475 Euclidean skeleton in 2D and 3D. Image Vis. Comput. 25 (10), 1519-1698.

Cronie, C., van Lieshout, M. N. M., 2016. Bandwidth selection for kernel estimators of the spatial intensity function.

URL https://arxiv.org/abs/1611.10221

Diggle, P. J., Fiksel, T., Grabarnik, P., Ogata, Y., Stoyan, D., T. M., 1994.

48о On Parameter Estimation for Pairwise Interaction Point Processes. Int. Stat. Rev. 62 (1), 99-117.

Ellis, S. P., 1986. A Limit Theorem for Spatial Point Processes. Adv. Appl. Probab. 18 (3), 646-659.

Gebäck, T., Marucci, M., Boissier, C., Arnehed, J., Heintz, A., 2015. Investigation of the Effect of the Tortuous Pore Structure on Water Diffusion through a Polymer Film Using Lattice Boltzmann Simulations. J. Phys. Chem. B 119, $5220-5227$.

Guan, Y., Sherman, M., Calvin, J. A., 2006. Assessing Isotropy for Spatial Point Processes. Biometrics 62 (1), 119-125. 
Häbel, H., Andersson, H., Olsson, A., Olsson, E., Larsson, A., Särkkä, A., 2016. Characterization of pore structure of polymer blended films used for controlled drug release. J. Control. Release 22, 151-158.

Heikkinen, J., Penttinen, A., 1999. Bayesian Smoothing in the Estimation of the Pair Potential Function of Gibbs Point Processes. Bernoulli 5 (6), 1119-1136.

Illian, J., Penttinen, A., Stoyan, H., Stoyan, D., 2008. Statistical Analysis and Modelling of Spatial Point Patterns. John Wiley \& Sons, Chichester.

Liebscher, A., Redenbach, C., 2013. Statistical Analysis of the Local Strut Thickness of Open Cell Foams. Image Anal. Stereol. 32 (1).

Marucci, M., Arnehed, J., Jarke, A., Matic, H., Nicholas, M., Boissier, C., von Corswant, C., 2013. Effect of the manufacturing conditions on the structure and permeability of polymer films intended for coating undergoing phase separation. Eur. J. Pharm. Biopharm. 83 (2), 301-306.

Marucci, M., Hjärtstram, J., Ragnarsson, G., Iselau, F., Axelsson, A., 2009. Coated formulations: New insights into the release mechanism and changes in the film properties with a novel release cell. J. Control. Release 136, 206212 .

Mateu, J., Montes, F., 2001. Likelihood Inference for Gibbs Processes in the Analysis of Spatial Point Patterns. Int. Stat. Rev. 69 (1), 81-104.

Mateu, J., Nicolis, O., 2012. Multiresolution analysis of spatial patterns to detect dominant directions. In: Working Papers GRASPA.

MAVI - Modular algorithms for volume images, 2015. Version (1.5.2). Fraunhofer ITWM, Kaiserslautern, Germany. URL http://www .mavi-3d.de/

Møller, J., Safavimanesh, F., Rasmussen, J. G., 2016. The cylindrical K-function 515 and Poisson line cluster point processes. Biometrika 103 (4), 937-954. 
Møller, J., Toftaker, H., 2014. Geometric anisotropic spatial point pattern analysis and cox processes. Scand. J. Stat. 41 (2), 414-435.

Møller, J., Waagepetersen, R. P., 2004. Statistical Inference and Simulation for Spatial Point Processes. Chapman \& Hall/CRC, Boca Raton.

Mugglestone, M., Renshaw, E., 1996. A practical guide to the spectral analysis of spatial point processes. Computational Statistics \& Data Analysis 21 (1), $43-65$.

Myllymäki, M., Mrkvička, T., Grabarnik, P., Seijo, H., Hahn, U., 2017. Global envelope tests for spatial processes. J. R. Stat. Soc. B.

${ }_{525}$ Ogata, Y., Tanemura, M., 1981. Estimation of interaction potentials of spatial point patterns through the maximum likelihood procedure. M. Ann. Inst. Stat. Math. 33 (1), 315-338.

Rajala, T., 2012. spatgraphs: Graphs for spatial point patterns. R package version 2.62 . URL http://CRAN.R-project.org/package=spatgraphs

Rajala, T., Särkkä, A., Redenbach, C., Sormani, M., 2016. Estimating geometric anisotropy in spatial point patterns. Spat. Stat. 15, 100-114.

Redenbach, C., Särkkä, A., Freitag, J., Schladitz, K., 2009. Anisotropy analysis of pressed point processes. AStA Adv. Stat. Anal. 93 (3), 237-261.

Safavimanesh, F., Redenbach, C., 2016. A comparison of functional summary statistics to detect anisotropy of three-dimensional point patterns.

URL https://arxiv.org/abs/1604.04211

Siegel, R. A., 2012. Porous Systems. In: Fundamentals and Applications of Controlled Release Drug Delivery. Springer, New York, pp. 29-251.

${ }_{540}$ Siepmann, F., Siepmann, J., Walther, M., MacRae, R. J., Bodmeier, R., 2008. Polymer blends for controlled release coatings. J. Control. Release 125 (1), $1-15$. 
Wen, H., Li, J. X., 2010. Introduction and overview of oral controlled release formulation design. In: Oral Controlled Release Formulation Design and Drug

${ }_{545}$ Delivery: Theory to Practice. John Wiley \& Sons, Hoboken, pp. 1-19.

Wong, K. Y., Chiu, S. N., 2016. Isotropy test for spatial point processes using stochastic reconstruction. Spat. Stat. 15, $56-69$.

Zhen, S., Davies, G. J., 1983. Calculation of the Lennard-Jones n-m potential energy parameters for metals. Phys. Status Solidi (a) 78 (2), 595-605. 